\title{
LOS DETERMINANTES DE LA LEGITIMIDAD GUBERNAMENTAL: EL SEXENIO DE ENRIQUE PENA NIETO
}

\section{THE DETERMINANTS OF GOVERNMENTAL LEGITIMACY; THE ENRIQUE PEÑA NIETO ADMINISTRATION}

\author{
LES FONDEMENTS DE LA LÉGITIMITÉ DU \\ GOUVERNEMENT SOUS LA PRÉSIDENCE \\ D'ENRIQUE PEÑA NIETO
}

\author{
Ma. Fernanda Somuano Ventura \\ El Colegio de México \\ fsomuano@colmex.mx
}

\begin{abstract}
Resumen: El objetivo de este estudio es explorar empíricamente los fundamentos de la legitimidad gubernamental durante la gestión de Enrique Peña Nieto. Para ello analizo, mediante datos individuales sobre percepciones y actitudes, qué determinó que los ciudadanos percibieran mayor o menor legitimidad en todo el gobierno (no sólo en el del presidente Peña Nieto) y qué factores influyeron en los cambios de dicha percepción. Con datos provenientes del Barómetro de las Américas (2012, 2014 y 2017) muestro que, tanto en 2014 como en 2017, la aprobación presidencial es el factor que tiene mayor influencia sobre la legitimidad gubernamental. Los ciudadanos que dijeron estar más satisfechos con las escuelas públicas y quienes se identificaron con el PRI también tendieron a percibir una mayor legitimidad gubernamental.

Palabras clave: legitimidad; Enrique Peña Nieto; aprobación presidencial; corrupción; México.
\end{abstract}

Aвstract: The objective of this study is to explore from an empirical perspective the grounds for governmental legitimacy during the administration of Enrique Peña Nieto. It uses individual perception and attitude data to analyze the factors that led citizens to perceive greater or lesser legitimacy in the government as a whole (not only that of President Peña Nieto) and which factors influence changes in this perception. With data taken from the Barómetro de las Américas (2012, 2014 and 2017), the paper shows that both in 2014 and in 
2017 presidential approval is the factor that has greatest influence on governmental legitimacy. Citizens who reported greater levels of satisfaction with public schools and who identified with the PRI party also tended to perceive greater governmental legitimacy.

Keywords: legitimacy; Enrique Peña Nieto; presidential approval; corruption; Mexico.

\section{Traducción de Gonzalo Celorio Morayta}

Résumé: L'article traite des fondements de la légitimité gouvernementale sous la présidence d'Enrique Peña Nieto. On examine des données individuelles concernant la vision et l'attitude des citoyens, afin d'établir ce qui les a menés à reconnaitre le président -et tout le gouvernement- comme légitime ou non, et pour quelles raisons ils auraient pu changer d'avis. L'information provenant du Barómetro de las Américas en 2012, 2014 et 2017 montre que, au cours des deux dernières années, le taux d'approbation du président est le facteur qui a l'incidence la plus profonde sur la légitimité du gouvernement dans son ensemble. Les citoyens qui ont manifesté le plus de satisfaction vis-àvis de l'école publique et ceux qui ont déclaré leur affinité avec le PRI avaient tendance aussi à percevoir le gouvernement comme étant légitime.

Mots clefs: Légitimité; Enrique Peña Nieto; taux d'approbation du président; corruption; Mexique.

\section{Traducción de BERNARdo Mabire}

Fecha de recepción: junio de 2019

Fecha de aceptación: noviembre de 2019 


\section{INTRODUCGIÓN}

H

asta hace unos años parecía que la democracia en México se había aceptado mayoritariamente como la mejor forma de gobierno. Según datos de LAPop de $2012,{ }^{1} 61.9 \%$ de los mexicanos opinaban que la democracia era mejor que cualquier forma de gobierno. No obstante, en 2014 esa cifra bajó a $58.7 \%$ y en 2017 a $46.6 \% .^{2}$ Otros indicadores de apoyo político, como la confianza en las instituciones y la satisfacción con la democracia, tampoco muestran resultados optimistas, pues revelan que la desafección y el descontento ciudadanos se han acrecentado de manera sostenida. ${ }^{3}$ De hecho, datos de la misma encuesta de 2017 muestran que casi la mitad de los mexicanos (46.4\%) desconfían de una institución fundamental para la democracia (los partidos políticos), y que sólo 3.6\% están satisfechos con el funcionamiento de la democracia.

La brecha entre la aspiración demócrata de los ciudadanos (medida a partir de qué tanto valoran los ideales democráticos y desprecian las alternativas autoritarias) y la percepción de la oferta democrática (medida a partir de la satisfacción con el desempeño democrático de su país) se conoce como déficit democrático. ${ }^{4}$ Por lo tanto, este concepto es aplicable a los casos en los que el desempeño de una democracia no es capaz de cumplir con las expectativas ciudadanas, ya sea una agencia gubernamental en específico, algunos de los arreglos institucionales que rigen al país o el régimen en su conjunto.

${ }^{1}$ Latin American Public Opinion Project (LAPOP), Universidad de Vanderbilt, https://www.vanderbilt.edu/lapop/

${ }^{2}$ La suma de 5, 6 y 7 en una escala de 1 a 7 , en donde 1 es muy en desacuerdo y 7 muy de acuerdo.

${ }^{3}$ Diana Guillén y Alejandro Monsiváis Carrillo (eds.), La legitimidad como desafío democrático. Expectativas públicas, capacidades institucionales y descontentos ciudadanos en México, México, El Colegio de la Frontera Norte, 2017.

${ }^{4}$ Pippa Norris, Democratic Deficit: Critical Citizens Revisited, Nueva York, Cambridge University Press, 2011. 
La razón por la cual este fenómeno es importante es que, aunque algunos optimistas afirman que la insatisfacción ciudadana puede servir como catalizador del activismo político $y$, por ende, fortalecer los procesos democratizadores, una mayoría de estudios apunta a que el descontento con el desempeño de las democracias provoca el distanciamiento cívico de los ciudadanos. Este hecho fomenta la erosión de modos de participación política convencionales y, peor aún, genera serios riesgos de inestabilidad y colapso en regímenes débiles que no tienen fundamentos sólidos de legitimidad en sus poblaciones. ${ }^{5}$

La legitimidad es la condición que proporciona justificación y consentimiento a quien ejerce el poder. Ésta, como cualidad que dota al poder de autoridad moral, ratifica el "derecho a gobernar" y motiva la cooperación política. ${ }^{6}$ Por ende, una cuestión fundamental para entenderla es identificar y analizar de dónde surge o qué la motiva.

El objetivo de este estudio es explorar empíricamente los fundamentos de la legitimidad gubernamental durante la gestión de Enrique Peña Nieto. Este caso es relevante porque involucra a un presidente que inició su mandato con una aprobación de $55 \%$, para caer cuatro años más tarde a menos de la mitad (25\% en noviembre de 2016, de acuerdo con datos de Buendía y Laredo), ${ }^{7}$ hasta llegar a $18 \%$ en agosto de 2018, logrando el peor nivel de aprobación para un presidente en los últimos 30 años (Consulta Mitofsky). ${ }^{8}$ Por lo tanto, es particularmente interesante explorar si esos niveles de aprobación y popularidad presidencial afectaron el apoyo más general al sistema político, es decir, la legitimidad.

De este modo, en el presente texto analizo, mediante datos individuales sobre percepciones y actitudes, qué determinó

${ }^{5}$ Ibid.

${ }^{6}$ Bruce Gilley, "The Meaning and Measure of State Legitimacy: Results for 72 Countries", European Journal of Political Research, 45, 3 (2006), pp. 499-525; Guillén y Monsiváis, op. cit.

${ }^{7}$ Buendía\&Laredo, https://www.buendiaylaredo.com/

${ }^{8}$ Mitofsky, http://www.consulta.mx/ 
que los ciudadanos percibieran mayor o menor legitimidad en todo el gobierno (no sólo en el del presidente Peña Nieto) y qué factores influyeron en los cambios de esta percepción. Es importante hacer énfasis en que la legitimidad gubernamental es distinta a la popularidad presidencial. Los datos que utilizo provienen del Barómetro de las Américas, correspondientes a los años 2012, 2014 y $2017 .^{9}$

\section{LOS FUNDAMENTOS DE LA LEGITIMIDAD}

El tema de la legitimidad ha sido recurrente en la historia del pensamiento político. Max Weber es quizá uno de los autores que vienen primero a la mente cuando se piensa en ese concepto y, aunque no define la legitimidad como tal, su trabajo permite inferir una noción del término. Primero, para Weber toda legitimidad está basada en determinadas valoraciones que, sin ser inalterables, tienen cierta permanencia temporal. Segundo, tales valoraciones o creencias tienen el carácter de verdaderas para una sociedad política. Tercera, la legitimidad otorga mayores probabilidades de estabilidad a las organizaciones. ${ }^{10}$

Un reto elemental de las democracias exitosas es la construcción de legitimidad de sus instituciones y procesos. Diversos académicos han desarrollado teorías para explicar la legitimidad del Estado, y el trabajo empírico para demostrar las consecuencias de dicha legitimidad ha sido menor. ${ }^{11}$ Estos autores han afirmado que el ejercicio del poder exige cierto grado de cooperación de aquellos sobre quienes se ejerce. La autoridad impuesta mediante la coerción o la violencia se

${ }^{9}$ El Barómetro de las Américas... (base de datos), 2012, 2014 y 2017, op. cit.

10 Max Weber, Economía y sociedad, México, FCE, 1964.

${ }^{11}$ David Easton, A Systems Analysis of Political Life, Nueva York, Wiley, 1965; Pierre Englebert, State legitimacy and development in Africa, Boulder, Lynne Rienne, 2002; Max Weber, "Legitimacy Politics and the State", en W. Conolly (ed.), Legitimacy and the State, Nueva York, University Press, 1984. 
cuestionará permanentemente y, por lo tanto, será inestable mientras no consiga generar lealtad y apoyo. La legitimidad entonces proporciona a la autoridad el consenso necesario para asegurar la obediencia de la población sin que sea preciso utilizar la fuerza. ${ }^{12}$

El análisis empírico de la legitimidad que hacen la ciencia política y la sociología contemporáneas se ha desarrollado en el campo delimitado justamente por la teoría de Weber, ${ }^{13}$ para quien la legitimidad está asociada con la probabilidad que tienen los tipos de dominación de generar acatamiento a un mandato determinado, basando su efectividad en la creencia acerca de las razones de dicha legitimidad. ${ }^{14}$

La idea de legitimidad puede utilizarse para referirse a líderes políticos, al régimen, al gobierno o al Estado. ${ }^{15}$ Lipset, por ejemplo, propone que "la legitimidad comporta la capacidad de un sistema político de generar y mantener la creencia de que las instituciones existentes son las más apropiadas para una sociedad". ${ }^{16}$ Por su parte, Easton la define como actitudes ciudadanas, específicamente "la convicción de que es correcto y propio obedecer a las autoridades y acatar las leyes por las cuales se rige el régimen". ${ }^{17}$ Parte de la importancia de analizar la legitimidad democrática permite estudiar las causas y consecuencias del apoyo que una sociedad manifiesta hacia la autoridad y el gobierno.

Sin embargo, como señala el propio Easton, dada su respetable historia como concepto central en la ciencia política, la legitimidad estatal todavía no recibe la atención que

12 Norberto Bobbio, El futuro de la democracia, México, FCE, 1986, p. 92; Guillén y Monsiváis, op. cit.

${ }^{13}$ Loc. cit.

${ }^{14}$ Max Weber, Ecomomía y sociedad, op. cit.

15 Bruce Gilley, “The Determinants of State Legitimacy: Results for 72 Countries”, International Political Science Review, 27, 1 (2006), pp. 47-71.

16 Seymour Martin Lipset, "Some Social Requisites of Democracy: Economic Development and Political Legitimacy", The American Political Science Review, 53, 1 (1959), p. 86.

17 David Easton, A Framework of Political Analysis, Englewood Cliffs, New Jersey, Prentice-Hall, 1965. 
merece en la investigación empírica. ${ }^{18}$ De hecho, la literatura que analiza los factores que moldean las percepciones que los individuos tienen sobre ésta es escasa.

Entender, desde el punto de vista teórico, las consecuencias de la legitimidad estatal es muy importante, pero analizarla como variable dependiente y explorar qué lleva a un ciudadano a confiar más o menos en el Estado, o a considerarlo más o menos legítimo es crucial. Estudiar las fuentes de legitimidad y confianza en el Estado, particularmente en contextos en que los legados autoritarios han deteriorado su reputación, puede ayudar a determinar las probabilidades de que el apoyo sea duradero o se resquebraje en el corto o mediano plazo. ${ }^{19}$

Así, podemos afirmar que el concepto de legitimidad es teóricamente muy rico, pero poco estudiado empíricamente, en especial porque el apoyo difuso al sistema es difícil de medir. ${ }^{20}$ Este apoyo es distinto de la popularidad de gobernantes o el apoyo a políticas específicas. La legitimidad es claramente un concepto multidimensional que en principio no puede capturarse por completo mediante una sola pregunta de una encuesta o cuestionario y, en general, no existe una medida aceptada para medirlo. David Beetham intenta capturar ese carácter al afirmar que se compone de tres dimensiones: las reglas que definen cómo se obtiene y ejerce el poder público, el reconocimiento público de la validez de esas reglas y la aceptación popular de su funcionamiento, y la expresión de acuerdo mediante comportamientos congruentes con las normas e instituciones prevalecientes. ${ }^{21}$

Otros estudios que han aceptado la multidimensionalidad del concepto han utilizado diferentes medidas de "legitimi-

18 Ibid.

${ }^{19}$ Danielle Carter, Sources of State Legitimacy in Contemporary South Africa: a Theory of Political Goods, Documento de trabajo núm. 134, Afrobarometer, 2011.

${ }^{20}$ Andrew Nathan, Political Culture and Diffuse Regime Support in Asia. Working Paper Series 43, Taipei, Asia Barometer Project Office, 2007, p. 3.

21 David Beetham, The Legitimation of Power, Basingstoke, Palgrave Macmillan, 2013. 
dad". En su estudio sobre modernización y cambio cultural, por ejemplo, Inglehart y Welzel lo miden como confianza institucional (evaluada en diversas instituciones gubernamentales). ${ }^{22}$ Otros estudios más recientes que también intentan calcularla, recurren a la medición de confianza o apoyo a las instituciones y van más allá al considerar también la satisfacción ciudadana con el régimen democrático. ${ }^{23}$

En su trabajo seminal sobre el concepto de apoyo político, Easton sugiere que es multidimensional y puede dividirse en dos categorías: "específico y difuso". El primero se refiere al tipo de evaluaciones ciudadanas relacionadas con las autoridades políticas y sus acciones. El segundo se refiere a características o aspectos del sistema. La legitimidad es un apoyo "difuso" 24 y, en cierto sentido, "refleja el hecho de que de una manera vaga o explícita [una persona] vea estos objetos [autoridades o régimen] como acordes con sus principios morales, su propio sentido de lo que es adecuado y correcto en la esfera política". ${ }^{25}$ Por ello, es razonable asumir que, cuando consideramos que un estado de cosas es moralmente correcto, es muy probable que lo juzguemos en términos muy favorables.

Easton propone tres tipos de fuentes que se asocian con la percepción que un individuo tiene sobre la legitimidad de una autoridad o régimen: 1) ideológicas, que se refieren a la convicción moral sobre la validez del régimen o la autoridad; 2) estructurales, respecto a la creencia en la validez de las estructuras y normas; 3) personales, tienen que ver con las características de las personas que fungen como autoridades. En este trabajo, me valgo de esta tipología para construir una medida de legitimidad gubernamental.

${ }^{22}$ Ronald Inglehart y Christian Welzel, Modernization, Cultural Change, and Democracy, New York, Cambridge University Press, 2005.

${ }^{23}$ Yun-han Chu et al., "Public Opinion and Democratic Legitimacy", Journal of Democracy, 19, 2 (2008), p. 77.

24 David Easton, "A Re-assessment of the Concept of Political Support”, British Journal of Political Science, 5, 4 (1975), pp. 435-457.

${ }^{25}$ Loc. cit. 


\section{LA VARIABLE DEPENDIENTE ¿CÓMO SE MIDE LA LEGITIMIDAD GUBERNAMENTAL?}

Partiendo de los tres tipos de fuentes de legitimidad propuestos por Easton, y con el fin de llevar el análisis al gobierno de Peña Nieto, decidí usar la valoración ciudadana de la democracia como mejor forma de gobierno y el orgullo por el sistema político como las medidas de la fuente ideológica de la legitimidad. Utilicé el respeto a las instituciones como la medida de la fuente estructural de la legitimidad y, finalmente, la confianza en el presidente para medir la fuente personal; en este último caso, asumiendo que los lazos de confianza se establecen, por lo general, con personas específicas (debido a las cualidades que muestran) y no con instituciones, que por definición son impersonales.

A continuación, presento la evolución de estos cuatro indicadores de 2012 a 2017. Como puede verse en el cuadro 1, los cuatro indicadores muestran una clara tendencia a la baja, y en algunos es francamente preocupante.

\section{CuAdro 1}

Evolución de indicadores relacionados con la legitimidad gubernamental (porcentajes)

\begin{tabular}{|l|r|r|r|}
\hline & 2012 & 2014 & 2017 \\
\hline Fuentes ideológicas & & & \\
\hline 1. Valoración de la democracia como mejor forma de gobierno & 24.4 & 25.9 & 14.4 \\
\hline 2. Orgullo por el sistema político & 13.3 & 9.5 & 9.2 \\
\hline Fuentes estructurales & & & \\
\hline 3. Respeto por las instituciones & 20.9 & 20.0 & 15.8 \\
\hline Fuentes personales & & & \\
\hline 4. Confianza en el presidente & 14.9 & 7.8 & 4.8 \\
\hline & & & \\
\hline
\end{tabular}

Fuente: LAPOP 2012, 2014 y 2017. 
El 2012 fue año de elecciones y de cambio de gobierno, pues el 1 de diciembre de ese año asumió la presidencia Enrique Peña Nieto. Aunque la elección mostró que la gente quería un cambio de rumbo en las políticas del país, Felipe Calderón terminó su periodo con alrededor de 53\% de aprobación presidencial (según datos de una poll de polls, promedio de diversas encuestas: Buendía y Laredo; Parametría; GEA-ISA; ${ }^{26}$ Consulta Mitofsky) y Enrique Peña comenzó con un porcentaje similar. En cambio, para 2014 ya era otra historia. Ese año coincide con dos situaciones que dañaron ampliamente la imagen del entonces presidente. El escándalo de la "Casa Blanca" (noviembre de 2014) y la desaparición de 43 estudiantes de la Escuela Normal de Ayotzinapa (septiembre de 2014). El primer caso devino en una investigación oficial por conflicto de interés, en el que un equipo de periodistas denunció la compra de una mansión de siete millones de dólares por Angélica Rivera -esposa del presidente- a una compañía de Grupo Higa, empresa con la que se habían tenido contratos de obra pública durante la gubernatura de Peña Nieto en el Estado de México entre 2005 y 2011. Aunque el presidente, su esposa y el secretario de Hacienda fueron exonerados, el resultado de la investigación fue muy cuestionado por ciudadanos y políticos de oposición y tuvo un impacto muy negativo sobre la popularidad del presidente.

El caso de los 43 estudiantes desaparecidos se refiere a una serie de episodios de violencia ocurridos la noche del 26 y la madrugada del 27 de septiembre de 2014, en los que la policía municipal y estatal de Iguala, Guerrero, persiguió y atacó a estudiantes de la Escuela Normal Rural de Ayotzinapa, a quienes acusaba de haber tomado un autobús de forma ilegal en la terminal local. En el ataque resultaron nueve personas fallecidas, 43 estudiantes desaparecidos y 27 heridos. La ineficacia de las autoridades para encontrar a los responsables de la desaparición de los normalistas, las contradiccio-

${ }^{26}$ Parametría, https://www.parametria.com.mx/; GEA-ISA, http:// structura.com.mx/gea 
nes en las que cayeron y las incongruencias de la investigación provocaron gran indignación en los padres de las víctimas y en la población en general, lo que a su vez generó fuerte desaprobación del desempeño del presidente y el gobierno en todos sus niveles.

Durante 2017 ocurrieron, asimismo, al menos dos escándalos de corrupción que estuvieron muy presentes durante varias semanas en la opinión pública. El primero estuvo relacionado con un socavón de ocho metros de diámetro y 16 de profundidad que ocasionó la muerte de dos personas en la autopista Cuernavaca-Ciudad de México. El sobrecosto de la obra de infraestructura que se acababa de inaugurar y la indiferencia con la que se recibieron las quejas de vecinos, que detectaron que un muro de la carretera estaba a punto de colapsar, hicieron que este caso generara gran indignación en la población.

El segundo caso fue la publicación de varias revelaciones que señalaban a Emilio Lozoya, exdirector de Pemex, como uno de los funcionarios que habían recibido sobornos millonarios de la constructora brasileña Odebrecht. Una segunda acusación sugería que el dinero que esta empresa había dado a Lozoya cuando trabajaba en el equipo de Peña Nieto había sido donado de manera ilegal a la campaña presidencial. En ese momento, Lozoya rechazó todas las acusaciones, pero el efecto en la opinión pública fue claramente muy negativo para el gobierno.

A partir de los cuatro indicadores incluidos en el cuadro, construí un índice de legitimidad gubernamental, que obtuve mediante un análisis de componentes principales. ${ }^{27}$ En el

${ }^{27}$ El análisis de componentes principales (ACP)es una técnica estadística que transforma de manera lineal un conjunto original de variables en un conjunto más pequeño de variables no correlacionadas que representan la mayor parte de información de las variables originales. El ACP es particularmente útil para reducir la dimensionalidad de un grupo de datos. Los primeros componentes principales describen la mayor parte de la varianza de los datos (más cuanto más correlacionadas estuvieran las variables originales). Véase George H. Dunteman, Principal Component Analysis, Newbury Park, Sage, 1989. 
caso del concepto que nos ocupa, el método permite reducir cuatro variables a una sola que las contiene. El primer componente extraído por este método fue el que utilicé como el índice de legitimidad (debido a que explica la mayor parte dela varianza del conjunto original de variables (50.08 para el índice de 2014 y $49.82 \%$ para el índice de 2017). Para comprobar la consistencia de los índices, hice una prueba de Alpha de Cronbach para cada año y obtuve un valor de .658 y .654 respectivamente, lo cual muestra que ambos índices tienen un grado de fiabilidad y congruencia interna suficiente (véase anexo 1).

\section{LOS DETERMINANTES DE LA LEGITIMIDAD GUBERNAMENTAL}

Como he mencionado anteriormente, hay pocos estudios empíricos que analicen los determinantes de la legitimidad gubernamental. La literatura existente parece coincidir en que son tres tipos de factores los que inciden en los niveles de legitimidad estatal: los atributos individuales de los ciudadanos, las actitudes, valores y experiencias de aquéllos en el nivel microsocial y la actuación o desempeño del gobierno y del sistema político en el nivel macro. Es más probable que los ciudadanos evalúen positivamente un gobierno más eficiente y un mejor desempeño económico. ${ }^{28}$ Se presupone, asimismo, que factores de largo plazo influyen en un tipo de apoyo más difuso, en cambio, los de corto plazo tienen un mayor efecto sobre un apoyo más específico. ${ }^{29}$

Siguiendo a Booth y Seligson, dividí los factores que determinan el grado de la legitimidad estatal en micro y macro-

${ }^{28}$ Goran Hayen y Michael Bratton (eds.), Governance and Politics in Africa, Boulder, Lynne Reinner Publishers, 1992; Larry Jay Diamond, The Spirit of Democracy: The Struggle to Build Free Societies Throughout the World, Nueva York, Henry Holt and Company, 2008.

${ }^{29}$ John A. Booth y Mitchell Seligson, The Legitimacy Puzzle in Latin America: Political Support and Democracy in Eight Nations, Nueva York, Cambridge University Press, 2009. 
sociales. ${ }^{30}$ Los primeros son exclusivos de los individuos e incluyen sus características socioeconómicas, demográficas, de actitudes y experiencias. Los determinantes macrosociales incluyen variables contextuales y de evaluación del desempeño, que forman las bases de las normas de la legitimidad que desarrollan los individuos. En este trabajo analicé ambos grupos de factores y desarrollé diversos modelos estadísticos para tratar de evaluar su impacto.

\section{Los factores microsociales}

Quizá porque la legitimidad de un sistema político se refiere primordialmente al aparato estatal o a un gobierno particular, y porque la mayoría de los estudios que se han hecho han considerado datos agregados a nivel nacional, se ha dado menos atención a características individuales demográficas y se ha puesto mayor énfasis en variables actitudinales y de cultura política. ${ }^{31}$ No obstante, variables como sexo, edad, religiosidad, educación o nivel socioeconómico es probable que incidan en el proceso de construcción o percepción de legitimidad y confianza de los individuos. Se puede pensar, por ejemplo, que los niveles de educación formal brindan mayores habilidades a los ciudadanos para evaluar las políticas implementadas por un régimen y que, por lo tanto, las percepciones sobre la legitimidad del régimen sean bajas si el desempeño gubernamental es deficiente. Al contrario, aquellos individuos que tienen menos educación formal pudieron haber asimilado mejor una educación nacionalista en la escuela y, por ende, tener menor capacidad para hacer un análisis crítico del gobierno y su trabajo. Por ejemplo, en Estados Unidos, Cole y sus coautores reportan que el apoyo al gobierno federal se incrementa de manera notable entre

31 Ibid. 
individuos blancos, republicanos, y graduados universitarios en $2002 .{ }^{32}$

En el caso del nivel socioeconómico, es plausible pensar que alguien con un alto nivel de recursos debiera generar una evaluación más positiva sobre la economía y las instituciones de un gobierno. Por el contrario, individuos menos favorecidos económicamente tenderán a una peor visión del desempeño gubernamental. Asimismo, es plausible que aquellos ciudadanos que reciben apoyos del gobierno lo perciban más legítimo que quienes no los reciben. Variables tales como el sexo o la edad también es probable que intervengan en las visiones y percepciones que los ciudadanos tienen sobre su gobierno.

En relación con la variable del sexo, es probable que las mujeres -al ser menos favorecidas por las políticas sociales que los hombres- tengan menores niveles de aprobación del gobierno y, por lo tanto, menos confianza en él. No obstante, hay estudios que muestran que las mujeres tienden a confiar más que los hombres en las personas e instituciones. ${ }^{33}$

En el caso de la edad, es probable que los ciudadanos más jóvenes se involucren menos en cuestiones políticas, porque su interés es menor. También es plausible que experimenten tasas más altas de desempleo que individuos mayores y reciban menores salarios que otros grupos de edad. Por lo tanto, es posible esperar que los jóvenes tengan peores evaluaciones

32 Richard L. Cole, John Kincaid y Andrew Parkin, "Public Opinion on Federalism in the United States and Canada in 2002: The Aftermath of Terrorism", Publius, 32, 4 (2002), p. 139. La revisión de la literatura revela que, en general, conforme mayor es el ingreso o el nivel de escolaridad de un individuo, menor es la confianza que tiene en el gobierno. Asimismo, varios estudios afirman que las mujeres tienden a confiar más que los hombres. Ver Najwa Sado Safadi y Margaret Lombe, "Exploring the Relationship Between Trust in Government and the Provision of Social Services in Countries of the Global South: The Case of Palestine, Journal of Social Service Research, 37,4 (2011), pp. 403-411.

${ }_{33}$ Instituto Federal Electoral (IFE) y El Colegio de México, Informe país sobre la calidad de la ciudadanía en México, México, IFE, El Colegio de México, 2014. 
y menos confianza en el gobierno y las instituciones que otros grupos.

Además de las variables sociodemográficas, es posible pensar que existen variables relacionadas con actitudes ciudadanas que pueden tener efecto en los niveles de confianza institucional. Estas variables son: la confianza interpersonal, que diversos estudios relacionan con la confianza institucional; la identificación partidista, porque es posible pensar que la identificación con un partido político puede influir en la confianza y apoyo del individuo al gobierno. Un individuo que se identifica con el partido gobernante tiene mayor probabilidad de apoyarlo que uno que se identifique con un partido de la oposición.

Recientemente ha habido estudios que muestran que la experiencia de los ciudadanos en su trato con instituciones gubernamentales o sus representantes (corrupción) o haber sido víctimas de algún delito pueden también ser factores que afecten su respaldo al gobierno. Evidentemente, quienes han vivido un episodio de corrupción que lo involucre, tenderán a una opinión más negativa de éste. ${ }^{34}$

\section{Los factores macrosociales}

Por último, como mencioné anteriormente, los factores macrosociales tienen que ver con variables del contexto en el que están inmersos los ciudadanos y con la evaluación de diversas facetas del quehacer gubernamental, tales como los servicios de educación y salud, por citar algunas. Es posible que el de-

${ }^{34}$ Ver Chad Kiewit de Jonge, "Corruption as a Mobilizing Grievance”, documento presentado en el congreso de la Latin American Studies Association, de 2009, Río de Janeiro, Brasil, 11 al 14 de junio de 2009; Regina Bateson, "Crime Victimization and Political Participation", The American Political Science Review, 106, núm. 3 (2012), pp. 570-583; Michelle Benson y Thomas Rochon, "Interpersonal Trust and the Magnitude of Protest. A Micro and Macro Level Approach”, Comparative Political Studies, 37, núm. 4 (2004), pp. 435-457. 
sempeño gubernamental tenga un efecto importante sobre las variables dependientes. Como mencioné anteriormente, existe todo un enfoque que relaciona el desencanto ciudadano con la democracia y la capacidad de los gobiernos de proveer servicios y bienes públicos de manera eficiente. Con un gobierno más eficiente y con mejor desempeño económico es más probable que los ciudadanos lo evalúen positivamente. ${ }^{35}$

\section{ANÁlisis ESTADÍSTICo}

Con el fin de encontrar qué factores son los que tienen mayor efecto sobre el índice de legitimidad gubernamental que construí, desarrollé dos modelos de regresión lineal, uno para 2014 y otro para 2017. Los datos provienen del Barómetro de las Américas. No incluí un tercer modelo para 2012 porque la encuesta se levantó en octubre de ese año, por lo que, aunque Peña Nieto ya era presidente electo, aún no asumía funciones. Con base en lo expuesto anteriormente, incluí variables sociodemográficas y otras que intentan representar factores micro y macrosociales. ${ }^{36}$

El modelo de 2014 muestra que la aprobación presidencial es la variable que tiene mayor efecto sobre el índice de legitimidad gubernamental; como era de esperarse, a mejor evaluación presidencial, la legitimidad del gobierno es mayor. De las otras tres variables que incluimos como factores macrosociales, una mayor satisfacción del ciudadano con las escuelas públicas también aumenta este índice. De acuerdo con lo esperado, una mayor percepción ciudadana de corrupción en los funcionarios públicos afecta de manera negativa la legitimidad gubernamental. En cambio, la satisfacción con los servicios de salud no presenta impacto estadísticamente significativo sobre la variable dependiente.

35 Goran Hayen y Michael Bratton (eds.), Governance and Politics in Africa, Boulder, Lynne Reinner Publishers. 1992; Larry Jay Diamond, The Spirit of Democracy... op. cit.

${ }^{36}$ El fraseo de las preguntas utilizadas para la construcción de variables se presenta en el anexo 2 . 


\section{Cuadro 2}

Determinantes de la legitimidad gubernamental (2014)

\begin{tabular}{|c|c|c|c|}
\hline & $B$ & Beta estándar & Sig. \\
\hline \multicolumn{4}{|l|}{ Factores microsociales } \\
\hline Sexo & $\begin{array}{c}.012 \\
(.050)\end{array}$ & .006 & .803 \\
\hline Edad & $\begin{array}{l}.004 * * \\
(.002)\end{array}$ & .057 & .039 \\
\hline Escolaridad & $\begin{array}{l}.003 \\
(.007)\end{array}$ & .012 & .678 \\
\hline Situación económica familiar & $\begin{array}{l}-.001 \\
(.035)\end{array}$ & -.001 & .984 \\
\hline Se identifica con el PRI & $\begin{array}{l}.214 * * * \\
(.074)\end{array}$ & .075 & .004 \\
\hline Confianza interpersonal & $\begin{array}{l}.096^{* * *} \\
(.030)\end{array}$ & .079 & .001 \\
\hline Policía solicitó soborno & $\begin{array}{l}-.179 * * * \\
(.067)\end{array}$ & -.072 & .008 \\
\hline Empleado público solicitó soborno & $\begin{array}{l}-.157^{*} \\
(.091)\end{array}$ & -.046 & .085 \\
\hline Soldado solicitó soborno & $\begin{array}{l}-.033 \\
(.206)\end{array}$ & -.004 & .871 \\
\hline Recibe apoyo gubernamental & $\begin{array}{c}.018 \\
(.063)\end{array}$ & .007 & .776 \\
\hline Víctima de algún delito en los últimos 12 meses & $\begin{array}{l}-.123 * * \\
(.058)\end{array}$ & -.053 & .036 \\
\hline \multicolumn{4}{|l|}{ Factores macrosociales } \\
\hline Percepción de corrupción en el gobierno & $\begin{array}{l}-.062 * * \\
(.030)\end{array}$ & -.051 & .043 \\
\hline Aprobación del desempeño del presidente & $\begin{array}{l}.407 * * * \\
(.026)\end{array}$ & .429 & .000 \\
\hline Satisfacción con escuelas públicas & $\begin{array}{l}.101^{* * *} \\
(.040)\end{array}$ & .069 & .011 \\
\hline Satisfacción con servicios de salud & $\begin{array}{c}.018 \\
(.035) \\
\end{array}$ & .014 & .610 \\
\hline Constante & $\begin{array}{c}-1.644 * * * \\
(.224)\end{array}$ & & .000 \\
\hline \multicolumn{4}{|l|}{$\mathrm{N}=1188$} \\
\hline $\mathrm{R}^{2}=.303$ & & & \\
\hline
\end{tabular}

$* \mathrm{p}<.10, * * \mathrm{p}<.05, * * * \mathrm{p}<.001$. Regresión lineal múltiple. 
De acuerdo con los resultados del modelo, entre los factores microsociales, la confianza interpersonal, seguida de la identidad partidista y la experiencia de intento de soborno por un policía son los factores que mayor efecto tienen sobre la legitimidad gubernamental. En el caso de los dos primeros, el impacto sobre ésta es positivo. El aumento en la confianza interpersonal y el hecho de identificarse con el PRI coincide con un aumento en este índice. El haber sufrido un intento de soborno por un policía, como era de esperarse, tiene el efecto contrario; haber sido víctima de algún delito o experimentado un intento de soborno de un empleado público también tienen un impacto negativo estadísticamente significativo sobre el índice de legitimidad gubernamental.

De las variables sociodemográficas, sólo la edad tiene un efecto sobre la variable dependiente. Conforme aumenta la edad de los ciudadanos es mayor la legitimidad percibida.

Los resultados para 2017 son similares a los obtenidos para 2014. En este modelo, dos factores de los que denominamos macrosociales tienen mayor efecto sobre el índice de legitimidad gubernamental. En este caso, los ciudadanos que tienen una percepción mayor de corrupción de los funcionarios públicos perciben que ésta es menor. En cuanto a la aprobación presidencial, ésta tiene una relación positiva con este índice: como era de esperarse, una mayor aprobación del presidente coincide con una mayor percepción de legitimidad en el gobierno.

Haber sido víctima de algún delito tiene una relación negativa con el índice de legitimidad, mientras que la satisfacción con las escuelas públicas, el identificarse con el PRI y la confianza interpersonal son los factores microsociales que tienen mayor efecto positivo sobre esta percepción. De las variables socioeconómicas, sólo la edad y la situación económica familiar afectan la impresión que los ciudadanos tienen sobre el gobierno: conforme aumenta la edad y mejora la situación económica familiar, los ciudadanos perciben una mayor legitimidad en el gobierno. 
Cuadro 3

Determinantes de la legitimidad gubernamental (2017)

\begin{tabular}{|c|c|c|c|}
\hline & $B$ & Beta estándar & Sig. \\
\hline \multicolumn{4}{|l|}{ Factores microsociales } \\
\hline Sexo & $\begin{array}{l}.002 \\
(.051)\end{array}$ & -.001 & .970 \\
\hline Edad & $\begin{array}{c}.003 * \\
(.002)\end{array}$ & .046 & .091 \\
\hline Escolaridad & $\begin{array}{l}-.005 \\
(.007)\end{array}$ & -.021 & .452 \\
\hline Situación económica familiar & $\begin{array}{l}.062^{* *} \\
(.031)\end{array}$ & .053 & .044 \\
\hline Se identifica con el PRI & $\begin{array}{l}.497 * * * \\
(.116)\end{array}$ & .106 & .000 \\
\hline Confianza interpersonal & $\begin{array}{l}.084 * * * \\
(.027)\end{array}$ & .077 & .002 \\
\hline Policía solicitó soborno & $\begin{array}{l}-.077 \\
(.067)\end{array}$ & -.034 & .252 \\
\hline Empleado público solicitó soborno & $\begin{array}{l}-.119 \\
(.087)\end{array}$ & -.039 & .175 \\
\hline Soldado solicitó soborno & $\begin{array}{c}.041 \\
(.167)\end{array}$ & .006 & .805 \\
\hline Recibe apoyo gubernamental & $\begin{array}{l}.112 * \\
(.060)\end{array}$ & .046 & .063 \\
\hline $\begin{array}{l}\text { Víctima de algún delito en los últimos } \\
12 \text { meses }\end{array}$ & $\begin{array}{l}-.309 * * * \\
(.054)\end{array}$ & -.148 & .000 \\
\hline \multicolumn{4}{|l|}{ Factores macrosociales } \\
\hline Percepción de corrupción en el gobierno & $\begin{array}{l}-.291 * * * \\
(.028)\end{array}$ & -.269 & .000 \\
\hline Aprobación desempeño del presidente & $\begin{array}{l}.097 * * * \\
(.016)\end{array}$ & .153 & .000 \\
\hline Satisfacción con escuelas públicas & $\begin{array}{l}.184^{* * * *} \\
(.037)\end{array}$ & .132 & .000 \\
\hline Satisfacción con servicios de salud & $\begin{array}{l}.079 * * * \\
(.033)\end{array}$ & .064 & .017 \\
\hline Constante & $\begin{array}{l}-.112 \\
(.247)\end{array}$ & & .651 \\
\hline \multicolumn{4}{|l|}{$\mathrm{N}=1226$} \\
\hline $\mathrm{R} 2=.279$ & & & \\
\hline
\end{tabular}

$* \mathrm{p}<.10, * * \mathrm{p}<.05, * * * \mathrm{p}<.001$. Regresión lineal múltiple. 


\section{DisCUSIÓN Y CONSIDERACIONES FINALES}

La legitimidad se ha definido tradicionalmente como un atributo del Estado que consiste en que una parte importante de su población considera que las leyes que la rigen son las correctas y las obedecen, sin que sea necesario el uso de la fuerza estatal.

La legitimidad es, según Easton, una forma de apoyo difuso que refleja sólidos lazos de lealtad o afecto hacia el sistema, a diferencia del apoyo específico que se refiere a la evaluación de las acciones de ciertas autoridades. ${ }^{37}$ Tras reconocer la multidimensionalidad de este concepto, en este capítulo traté de analizar los factores que afectan las percepciones ciudadanas sobre la legitimidad gubernamental en México, medida con un indicador compuesto por cuatro variables: valoración de la democracia como mejor forma de gobierno, sentimiento de orgullo por el sistema político, respeto por las instituciones y acuerdo con el sistema legal y la valoración de la democracia.

Con datos de la Encuesta LAPOP (2014 y 2017) construí dos modelos multivariados que mostraron que, en ambos años, una combinación de factores macrosociales y microsociales determina la percepción que tienen los ciudadanos sobre la legitimidad gubernamental. Es interesante comprobar que la legitimidad tiene distintas dimensiones y diversos determinantes en distintos niveles de análisis. Es decir, las percepciones de los ciudadanos sobre la legitimidad del Estado dependen de sus características y actitudes individuales, pero también de elementos contextuales relacionados con la evaluación de las tareas de los gobiernos.

Los datos muestran que, en ambos años, la aprobación presidencial es el factor con mayor influencia sobre la legitimidad gubernamental. Los ciudadanos que dijeron estar más satisfechos con las escuelas públicas y quienes se identificaron con el PRI también tendieron a percibir que ésta

${ }^{37}$ David Easton, “A Re-assessment...”, art. cit. 
es mayor. Por el contrario, quienes tuvieron una experiencia de intento de soborno por un policía, quienes observaron mayores niveles de corrupción entre los empleados públicos o quienes fueron víctimas de un delito tendieron a evaluar de peor manera la legitimidad del gobierno. El impacto de la percepción ciudadana de la corrupción fue especialmente importante en 2017, muy probablemente por todos los escándalos que se fueron acumulando en el sexenio de Peña Nieto. De acuerdo con las expectativas, los jóvenes mostraron peores evaluaciones de la legitimidad gubernamental que otros grupos etarios en ambos años. Es decir, la percepción de ésta depende no solamente de las experiencias personales de los ciudadanos (que pueden incluir su interacción con funcionarios públicos), sino también de apreciación general de cómo el gobierno realiza su trabajo.

El hecho de que la legitimidad dependa de manera importante del desempeño del gobierno claramente tiene ventajas. Un buen desempeño generará mayores niveles de legitimidad para el régimen. Los resultados que obtuve indican que el desempeño del gobierno en relación con la educación pública es lo que más valoran los ciudadanos. Sin embargo, lo contrario también es cierto. Este estudio prueba cómo los escándalos de corrupción y la ineficacia del gobierno tuvieron un claro efecto negativo en el índice de legitimidad durante el sexenio de Peña Nieto. Además, muestra empíricamente que el nivel de legitimidad se ve influido por la evaluación que los ciudadanos hacen de agencias estatales específicas encargadas de educación, salud y seguridad pública. La pregunta que queda sin respuesta es: ¿¿n qué momento la insatisfacción por el desempeño de los gobernantes o autoridades específicas se convierte en ilegitimidad del régimen? Responder esta pregunta es un desafío para una nueva investigación. 


\section{BibLIOGRAFÍA}

Bateson, Regina, "Crime Victimization and Political Participation", The American Political Science Review, 106, núm. 3 (2012), pp. 570-583.

Beetham, David, The Legitimation of Power, Basingstoke, Palgrave Macmillan, 2013.

Behrend, Jaqueline, "The Uneveness of Democracy at the Subnational Level: Provincial Closed games in Argentina", Latin American Research Review 46, 1 (2011), pp. 150-176.

Benson, Michelle y Thomas Rochon, "Interpersonal Trust and the Magnitude of Protest. A Micro and Macro Level Approach", Comparative Political Studies, 37, núm. 4 (2004), pp. 435-457.

Bobbio, Norberto, El futuro de la democracia, México, FCE, 1986.

Воотн, John A. y Mitchell Seligson, The Legitimacy Puzzle in Latin America: Political Support and Democracy in Eight Nations, Nueva York, Cambridge University Press, 2009.

Bunce, Valerie, "Comparative Democratization. Big and Bounded Generalizations”, Comparative Political Studies, 33, 6-7 (2000), pp. 703-734.

Carter, Danielle, Sources of State Legitimacy in Contemporary South Africa: a Theory of Political Goods, documento de trabajo núm. 134 de Afrobarometer, 2011.

Chanley, Virginia A., Thomas J. Rudolph y Wendy M. Rahn, "The Origins and Consequences of Public Trust in Government: A Time-Series Analysis", Public Opinion Quarterly, 64, 3 (2000), pp. 239-256.

Chu, Yun-han, Michael Bratton, Marta Lagos, Sandeep Shastri y Mark Tessler, "Public Opinion and Democratic Legitimacy", Journal of Democracy, 19, 2 (2008), pp. 74-87.

Cole, Richard L., John Kincaid y Andrew Parkin, "Public Opinion on Federalism in the United States and Canada in 2002: The Aftermath of Terrorism", Publius, 32, 4 (2002), pp.123-148.

Diamond, Larry Jay, The Spirit of Democracy: The Struggle to Build Free Societies Throughout the World, Nueva York, Henry Holt and Company, 2008. 
Dincecco, Mark y Gabriel Katz, "State Capacity and Long run Economic Performance”, Economic Journal, 126, 590 (2016), pp. 189-218.

Dunteman, George H., Principal Component Analysis, Newbury Park, Sage, 1989.

DurAzo, Julián, "Neo-Patrimonilaism and Subnational Authoritarianism in Mexico: The case of Oaxaca", Journal of Politics in Latin America, 2 (2010), pp. 85-113.

Easton, David, A Framework of Political Analysis, Englewood Cliffs, Prentice-Hall, 1965.

Easton, David, A Systems Analysis of Political Life, Nueva York, Wiley, 1965.

Easton, David, "A Re-assessment of the Concept of Political Support”, British Journal of Political Science, 5, 4 (1975), pp. 435-457.

El Barómetro de las Américas por el Proyecto de Opinión Pública de América Latina (LAPOP), México, 2012 (base de datos en línea), www.LapopSurveys.org

Englebert, Pierre, State legitimacy and development in Africa, Boulder, Lynne Rienne, 2002.

FJelde, Hanne e Indra de Soysa, "Coercion, Co-optation, or Cooperation?: State Capacity and the Risk of Civil War, 1961-2004", Conflict Management and Peace Science, 26, 1 (2009), pp. 5-25.

Geddes, Barbara, Politician's Dilemma: Building State Capacity in Latin America, Berkeley, University of California Press, 1994.

Gervasoni, Carlos, "Democracia, Autoritarismo e Hibridez en las Provincias Argentinas: La Medición y Causas de los Regímenes Subnacionales", Journal of Democracy en Español, 3 (2011), pp. 75-93.

Gervasoni, Carlos, "Poliarquía a Nivel Subnacional. Aspectos Conceptuales y Normativos en el Contexto de las Democracias Federales", Colección, 16 (2005), pp. 83-122.

Gibson, Edward L., Boundary Control. Subnational Authoritarianism in Federal Democracies, Cambridge, University Press, 2012.

Gilley, Bruce, "The Determinants of State Legitimacy: Results for 72 Countries”, International Political Science Review, 27, 1 (2006), pp. $47-71$. 
Gilley, Bruce, "The Meaning and Measure of State Legitimacy: Results for 72 Countries", European Journal of Political Research, 45, 3 (2006), pp. 499-525.

Giraudy, Agustina, "Conceptualizing State Strength: Moving Beyond Strong and Weak States", Revista de Ciencia Politica, 32, 3 (2012), pp. 599-611.

Guillén, Diana y Alejandro Monsiváis Carrillo (eds.), La legitimidad como desafío democrático. Expectativas públicas, capacidades institucionales y descontentos ciudadanos en México, México, El Colegio de la Frontera Norte, 2017.

Hayen, Goran y Michael Bratton (eds.), Governance and Politics in Africa, Boulder, Lynne Reinner Publishers, 1992.

Huber, Evelyne, Dietrich Rueschemeyer y John D. Stephens, "The Impact of Economic Development on Democracy", The Journal of Economic Perspectives, 7, 3 (1993), pp. 71-86.

Inglehart, Ronald y Christian Welzel, Modernization, Cultural Change, and Democracy, Nueva York, Cambridge University Press, 2005.

Instituto Federal Electoral (IFE) y El Colegio de México, Informe país sobre la calidad de la ciudadanía en México, México, IFE, El Colegio de México, 2014, http:/ /www.ine.mx/archivos2/s/DECEYEC/ EducacionCivica/Informe_pais_calidad_ciudadania_IFE_FI NAL.pdf

Kaufman, Daniel, Aart Kraay y Pablo Zoiso, Governance Matters, World Bank, Policy Research Working Paper núm. 2196, 1999. Kiewit De Jonge, Chad, "Corruption as a Mobilizing Grievance", documento presentado en el congreso de la Latin American Studies Association de 2009, Río de Janeiro, Brasil, 11 al 14 de junio de 2009.

KurTz, Marcus, Latin American State Building in Comparative Perspective, Nueva York; Cambridge University Press, 2013.

Kurtz, Marcus J. y Andrew Schrank, "Capturing State Strength: Experimental and Economic Approaches”, Revista de Ciencia Política, 323 (2012), pp. 613-621.

Linz, Juan y Alfred StePan, Problems of Democratic Transition and Consolidation: Southern Europe, South America and Post-Communist Europe, Baltimore, Johns Hopkins University Press, 1996. 
LiPset, Seymour Martin, "Some Social Requisites of Democracy: Economic Development and Political Legitimacy", The American Political Science Review, 53, 1 (1959), pp. 69-105.

LunA, Juan Pablo y Sergio Toro, "Capacidad del Estado y gobernabilidad democrática en América Latina: un acercamiento de medición y evaluación basado en el Barómetro de las Américas", Perspectivas desde el Barómetro de las Américas 2014, 102 (2014), pp.1-6.

Mann, Michael, "The Autonomous Power of the State: its origins, mechanisms and results", European Journal of Sociology, 25 (1984), pp. 185-213.

Mann, Michael, The Sources of Political Power, New York, Cambridge University Press, 1986.

Nathan, Andrew, Political Culture and Diffuse Regime Support in Asia. Working Paper Series 43, Taipei, Asia Barometer Project Office, 2007.

Norris, Pippa, Democratic Deficit: Critical Citizens Revisited, Nueva York, Cambridge University Press, 2011.

Programa de las Naciones Unidas para el Desarrollo, Informe sobre desarrollo humano 2004, Madrid, México, PNUD-Mundi-Prensa, 2004, http://hdr.undp.org/sites/default/files/hdr_2004_ es.pdf

Przeworski, Adam, Sustainable Democracy, Cambridge, University Press, 1995.

Roberts, Cynthia y Thomas Sherlock, "Bringing the Russian state back in: Explanations of the Derailed Transition to Market Democracy", Comparative Politics, 31, 4 (1999), pp. 477-498.

SAfadi, Najwa Sado y Margaret Lombe, "Exploring the Relationship Between Trust in Government and the Provision of Social Services in Countries of the Global South: The Case of Palestine”, Journal of Social Service Research, 37, 4 (2011), pp. 403-411.

SoIfER, Hillel, "Measuring State Capacity in Contemporary Latin America”, Revista de Ciencia Política, 32, 3 (2012), pp. 585-598.

SoIfer, Hillel y Matthias Vom Hau, "Unpacking the Strength of the State: The Utility of State Infrastructural Power", Studies in Comparative International Development, 43, núm. 3-4 (2008), pp. 219-230. 
Tiluy, Charles, Coercion, Capital and the European States, A.D. 9901990, Cambridge, Basil Blackwell, 1990.

Weber, Max, "Legitimacy Politics and the State", en W. Conolly (ed.), Legitimacy and the State, Nueva York, New York University Press, 1984.

Weber, Max, Ecomomía y sociedad, México, FCE, 1964. 


\section{Anexo 1}

\section{Escala para 2014}

\begin{tabular}{|c|c|c|}
\hline \multicolumn{3}{|c|}{ Estadísticos de fiabilidad } \\
\hline $\begin{array}{c}\text { Alfa de } \\
\text { Cronbach }\end{array}$ & $\begin{array}{c}\text { Alfa de Cronbach } \\
\text { basada en los elementos } \\
\text { tipificados }\end{array}$ & N de elementos \\
\hline .658 & .652 & 4 \\
\hline
\end{tabular}

\section{Matriz de correlaciones interelementos}

\begin{tabular}{|l|c|c|c|c|}
\hline & $\begin{array}{c}\text { Confianza en } \\
\text { el ejecutivo }\end{array}$ & $\begin{array}{c}\text { Orgullo por } \\
\text { el sistema }\end{array}$ & $\begin{array}{c}\text { Democracia como } \\
\text { la mejor forma de } \\
\text { gobierno }\end{array}$ & $\begin{array}{c}\text { Respeto } \\
\text { institucional }\end{array}$ \\
\hline Confianza en el ejecutivo & 1.000 & .498 & .200 & .361 \\
\hline Orgullo por el sistema & .498 & 1.000 & .181 & .477 \\
\hline $\begin{array}{l}\text { Democracia como la mejor } \\
\text { forma de gobierno }\end{array}$ & .200 & .181 & 1.000 & .197 \\
\hline Respeto institucional & .361 & .477 & .197 & 1.000 \\
\hline
\end{tabular}

\begin{tabular}{|c|c|c|c|}
\hline \multicolumn{4}{|c|}{ Estadísticos de la escala } \\
\hline Media & Varianza & Desviación típica & N de elementos \\
\hline 16.8677 & 26.000 & 5.09900 & 4 \\
\hline
\end{tabular}

\section{Escala para 2017}

\begin{tabular}{|c|c|c|}
\hline \multicolumn{3}{|c|}{ Estadísticos de fiabilidad } \\
\hline $\begin{array}{c}\text { Alfa de } \\
\text { Cronbach }\end{array}$ & $\begin{array}{c}\text { Alfa de Cronbach } \\
\text { basada en los elementos } \\
\text { tipificados }\end{array}$ & N de elementos \\
\hline .654 & .648 & 4 \\
\hline
\end{tabular}




\section{Matriz de correlaciones interelementos}

\begin{tabular}{|l|c|c|c|c|}
\hline & $\begin{array}{c}\text { Respeto } \\
\text { institucional }\end{array}$ & $\begin{array}{c}\text { Orgullo por el } \\
\text { sistema politico }\end{array}$ & $\begin{array}{c}\text { Confianza en } \\
\text { el ejecutivo }\end{array}$ & $\begin{array}{c}\text { Democracia como } \\
\text { la mejor forma de } \\
\text { gobierno }\end{array}$ \\
\hline Respeto institucional & 1.000 & .451 & .326 & .212 \\
\hline $\begin{array}{c}\text { Orgullo por el sistema } \\
\text { politico }\end{array}$ & .451 & 1.000 & .539 & .182 \\
\hline Confianza en el ejecutivo & .326 & .539 & 1.000 & .182 \\
\hline $\begin{array}{c}\text { Democracia como la } \\
\text { mejor forma de gobierno }\end{array}$ & .212 & .182 & .182 & 1.000 \\
\hline
\end{tabular}

\section{Estadísticos de la escala}

\begin{tabular}{|c|c|c|c|}
\hline Media & Varianza & Desviación típica & $N$ de elementos \\
\hline 14.5788 & 28.552 & 5.34341 & 4 \\
\hline
\end{tabular}

\section{Anexo 2}

\section{Variables con las que se construyó el índice de legitimidad gubernamental}

\begin{tabular}{|l|l|l|}
\hline \multicolumn{1}{|c|}{ Nombre de la variable } & \multicolumn{1}{|c|}{ Pregunta } & \multicolumn{1}{c|}{ Codificación } \\
\hline $\begin{array}{l}\text { Respeto por las } \\
\text { instituciones del país }\end{array}$ & $\begin{array}{l}\text { ¿Hasta qué punto tiene usted respe- } \\
\text { to por las instituciones políticas de } \\
\text { México? }\end{array}$ & $\begin{array}{l}\text { Escala del } 1 \text { al } 7 \text { en donde 1 } \\
\text { es nada y } 7 \text { es mucho }\end{array}$ \\
\hline $\begin{array}{l}\text { Sentimiento de } \\
\text { orgullo por el sistema } \\
\text { político }\end{array}$ & $\begin{array}{l}\text { ¿Hasta qué punto se siente usted or- } \\
\text { gulloso de vivir bajo el sistema políti- } \\
\text { co de México? }\end{array}$ & $\begin{array}{l}\text { Escala del } 1 \text { al } 7 \text { en donde 1 } \\
\text { es nada y } 7 \text { es mucho }\end{array}$ \\
\hline $\begin{array}{l}\text { Confianza en el } \\
\text { ejecutivo }\end{array}$ & $\begin{array}{l}\text { ¿Hasta qué punto tiene confianza en } \\
\text { el presidente? }\end{array}$ & $\begin{array}{l}\text { Escala del } 1 \text { al } 7 \text { en donde 1 } \\
\text { es nada y } 7 \text { es mucho }\end{array}$ \\
\hline $\begin{array}{l}\text { Valoración de la } \\
\text { democracia como } \\
\text { la mejor forma de } \\
\text { gobierno }\end{array}$ & $\begin{array}{l}\text { Puede que la democracia tenga pro- } \\
\text { blemas, pero es mejor que cualquier } \\
\text { otra forma de gobierno. ¿Hasta qué } \\
\text { punto está de acuerdo o en desacuer- } \\
\text { do con esta frase? }\end{array}$ & $\begin{array}{l}\text { Escala del } 1 \text { al } 7 \text { en donde } \\
\text { es muy en desacuerdo y } 7 \\
\text { muy de acuerdo }\end{array}$ \\
\hline
\end{tabular}




\section{Variables independientes/factores microsociales y macrosociales}

\begin{tabular}{|c|c|c|}
\hline Nombre de la variable & Pregunta & Codificación \\
\hline Sexo & Sexo & $\begin{array}{l}1=\text { hombre } \\
2=\text { mujer }\end{array}$ \\
\hline $\begin{array}{l}\text { Situación económica } \\
\text { en el hogar }\end{array}$ & $\begin{array}{l}\text { El salario o sueldo que usted recibe y } \\
\text { el total del ingreso de su hogar: }\end{array}$ & $\begin{array}{l}\text { 1= no les alcanza bien y } \\
\text { tienen grandes dificultades } \\
\text { 2=no les alcanza y tienen } \\
\text { dificultades } \\
\text { 3=les alcanza justo sin } \\
\text { grades dificultades } \\
\text { 4=les alcanza bien y } \\
\text { pueden ahorrar }\end{array}$ \\
\hline $\begin{array}{l}\text { Identidad partidista } \\
\text { priista }\end{array}$ & $\begin{array}{l}\text { ¿En este momento simpatiza con al- } \\
\text { gún partido político? ¿Con cuál? }\end{array}$ & $\begin{array}{l}1=\text { PRI } \\
0=\text { todos los demás }\end{array}$ \\
\hline $\begin{array}{l}\text { Confianza } \\
\text { interpersonal }\end{array}$ & $\begin{array}{l}\text { Ahora hablemos de la gente de por } \\
\text { aquí, ¿diría que la gente de su comun- } \\
\text { idad es muy confiable, algo confiable, } \\
\text { poco confiable o nada confiable? }\end{array}$ & $\begin{array}{l}1=\text { nada confiable } \\
2=\text { poco confiable } \\
3=\text { algo confiable } \\
4=\text { muy confiable }\end{array}$ \\
\hline $\begin{array}{l}\text { Víctima de algún } \\
\text { delito en los últimos } \\
\text { doce meses }\end{array}$ & $\begin{array}{l}\text { ¿Ha sido usted víctima de algún acto } \\
\text { de delincuencia en los últimos } 12 \text { me- } \\
\text { ses? Es decir, dha sido usted víctima } \\
\text { de un robo, hurto, agresión, fraude, } \\
\text { chantaje, extorsión, amenazas o al- } \\
\text { gún otro tipo de acto delincuencial } \\
\text { en los últimos } 12 \text { meses? }\end{array}$ & $\begin{array}{l}\text { Sí }=1 \\
\text { No=0 }\end{array}$ \\
\hline $\begin{array}{l}\text { Policía solicitó } \\
\text { soborno }\end{array}$ & $\begin{array}{l}\text { ¿Algún agente de la policía le pidió } \\
\text { una mordida (o soborno) en los últi- } \\
\text { mos } 12 \text { meses? }\end{array}$ & $\begin{array}{l}\mathrm{Sí}=1 \\
\mathrm{No}=0\end{array}$ \\
\hline $\begin{array}{l}\text { Empleado público } \\
\text { solicitó soborno }\end{array}$ & $\begin{array}{l}\text { ¿Algún empleado público le pidió } \\
\text { una mordida (o soborno) en los últi- } \\
\text { mos } 12 \text { meses? }\end{array}$ & $\begin{array}{l}\mathrm{Sí}=1 \\
\mathrm{No}=0\end{array}$ \\
\hline $\begin{array}{l}\text { Soldado solicitó } \\
\text { soborno }\end{array}$ & $\begin{array}{l}\text { ¿En los últimos doce meses algún sol- } \\
\text { dado u oficial militar le ha solicitado } \\
\text { un soborno o mordida? }\end{array}$ & $\begin{array}{l}\mathrm{Sí}=1 \\
\mathrm{No}=0\end{array}$ \\
\hline $\begin{array}{l}\text { Percepción sobre } \\
\text { corrupción en el } \\
\text { gobierno }\end{array}$ & $\begin{array}{l}\text { Pensando en los políticos de México, } \\
\text { ¿cuántos de ellos cree usted que es- } \\
\text { tén involucrados en corrupción? }\end{array}$ & $\begin{array}{l}\text { 1= ninguno } \\
2=\text { menos de la mitad } \\
3=\text { la mitad de los políticos } \\
4=\text { más de la mitad } \\
5=\text { todos }\end{array}$ \\
\hline
\end{tabular}




\begin{tabular}{|c|c|c|}
\hline $\begin{array}{l}\text { Recibe apoyo } \\
\text { gubernamental }\end{array}$ & $\begin{array}{l}\text { ¿Usted o alguien en su casa recibe } \\
\text { ayuda del gobierno, regular o periódi- } \\
\text { ca, en dinero, alimento o en produc- } \\
\text { tos, sin contar las pensiones? }\end{array}$ & $\begin{array}{l}\mathrm{Sí}=1 \\
\mathrm{No}=0\end{array}$ \\
\hline $\begin{array}{l}\text { Satisfacción con las } \\
\text { escuelas públicas }\end{array}$ & $\begin{array}{l}\text { Y pensando en la ciudad donde usted } \\
\text { vive, ¿qué tan satisfecho está con la } \\
\text { calidad de las escuelas públicas? }\end{array}$ & $\begin{array}{l}1=\text { muy insatisfecho } \\
2=\text { insatisfecho } \\
3=\text { satisfecho } \\
\text { 4=muy satisfecho }\end{array}$ \\
\hline $\begin{array}{l}\text { Satisfacción con los } \\
\text { servicios de salud }\end{array}$ & $\begin{array}{l}\text { ¿Qué tan satisfecho está con la cali- } \\
\text { dad de los servicios médicos y de } \\
\text { salud públicos? }\end{array}$ & $\begin{array}{l}1=\text { muy insatisfecho } \\
2=\text { insatisfecho } \\
3=\text { satisfecho } \\
\text { 4=muy satisfecho }\end{array}$ \\
\hline $\begin{array}{l}\text { Aprobación } \\
\text { presidencial }\end{array}$ & $\begin{array}{l}\text { Hablando en general acerca del go- } \\
\text { bierno actual, ¿cómo diría usted que } \\
\text { es el trabajo que está realizando el } \\
\text { presidente Enrique Peña Nieto? }\end{array}$ & $\begin{array}{l}1=\text { pésimo } \\
2=\text { malo } \\
3=\text { regular } \\
4=\text { bueno } \\
5=\text { muy bueno }\end{array}$ \\
\hline
\end{tabular}

